\title{
Need of Quantum mechanics
}

\author{
Bishow Raj Tiwari \\ IOE, Paschimanchal Campus, Pokhara,Email: tiwari.br@yahoo.com
}

Physics in which most of the physical phenomena can be successfully described by using Newton's laws of motion is called classical physics. In fact, it includes the classical mechanics \& the electromagnetic theory. In classical mechanics, the matter is regarded as consisting of point particles which move under the action of mutually interacting forces. The most important law in classical mechanics is the law of motion i.e., Force $=$ mass $\times$ acceleration. Law explained successfully the motion of the planets as well as many other electrically neutral macroscopic bodies. On the other hand, the electromagnetic theory explained the physical phenomena concerning magnetism \& electricity in terms of electric \& magnetic fields. Maxwell concluded that both electric \& magnetic field travel in free space as waves which carry energy. Hence, matter consists of point particles carrying mass and radiation consisting of waves carrying energy $\&$ momentum.

The laws of mechanics started by Newton and their development by Lagrange, Poisson, Jacob, Hamilton, Maxwell's equations in electrodynamics etc. seemed perfect universal \& gave satisfactory description of the electromagnetic phenomena. Up to the end of the nineteenth century, the classical physics was so successful $\&$ impressive in explaining physical phenomena that the scientists of that time absolutely believed that they were capable of explaining all physical phenomena.

However, with the discoveryofelectronasafundamental particle by J.J. Thomson in 1897, most of the studies in physics were concentrated to explore microscopic or atomic systems which are beyond the scope of direct observation. Many difficulties were encountered with phenomena such as spectral distribution of heat radiation from black body, specific heats of solids at low temperature etc. In addition, the laws of classical physics could not explain phenomena involving very high velocity \& the phenomena involving very short distances like atoms and other microscopic particles. The first indication of inadequacy of the classical physics was seen in the beginning of the twentieth century when it could not explain experimentally observed spectra of a blackbody radiations which was resolved by max Planck in 1900. This in turn led to the introduction of the quantum theory.

According to Classical physics, the exchange of energy between radiation and matter is continuous. According to Rayleigh-Jeans law for the blackbody radiation, the light of frequency $u$ can give up any amount of energy on absorption which is given by:

$\mathrm{u}(\mathrm{v}) \mathrm{d} v=\left(\frac{8 \pi \mathrm{v}^{2}}{\mathrm{c}^{3}} \mathrm{dv}\right) \mathrm{KT}^{\circ}$

where, $u(v)$ is the energy per unit volume with frequency between $v \& v+d v, K$ is the Bottzmann Constant, $\mathrm{T}^{\circ}$ the enclosure temperature and $\mathrm{c}$ the velocity of light. The equation (i) gives the finite value only at low frequency but when $v \rightarrow \infty$,

$\int_{0}^{\infty} u(v) d v=\frac{8 \pi \mathrm{KT}}{\mathrm{C}^{3}} \int_{0}^{\infty} \mathrm{v}^{2} \mathrm{~d} v=\infty$

To resolve the Paradax, Planck introduced the hypothesis of the quantization of energy i.e., the radiation of frequency $(v)$ can only exchange energy with mater in discrete form, $\mathrm{E}=\mathrm{h} \mathrm{v}, \mathrm{h}=6.626 \times 10^{-34}$ JS or $6.626 \times 10^{-27}$ ergs is a Planck's Constant. Such discrete units are tiny packets, called quantum. Then the only available energies for the wave of frequency $v$ are; $o, h v, 2 h v, \ldots . . . n h v,(n+1) h v \ldots .$. . The probability of having energy nh $v$ at temperature $\mathrm{T}^{\circ}$ is given by the boltzmann factor $\exp \left[-n h \mathrm{v} /\left(\mathrm{KT}^{\circ}\right)\right]$. On Planck's hypothesis, the mean energy in the wave of frequency $v$ is ,

$$
\begin{aligned}
& \frac{\mathrm{hu} \sum_{\mathrm{n}=0}^{\infty} \mathrm{n} \exp \left[-\mathrm{nhv}\left[\left(\mathrm{KT}^{\circ}\right)\right]\right.}{\sum_{\mathrm{n}=0}^{\infty} \exp \left[-\mathrm{nh} v\left[\left(\mathrm{KT}^{\circ}\right)\right]\right.}=\frac{\mathrm{hu}}{\exp \left[\mathrm{hu}\left[\left(\mathrm{KT}^{\circ}\right)\right]-1\right.} \\
& \mathrm{u}(\mathrm{v})=\frac{8 \pi \mathrm{h} \mathrm{v}^{3}}{\mathrm{C}^{3}} \frac{1}{\exp \left[\mathrm{hu}\left[\left(\mathrm{KT}^{\circ}\right)\right]-1\right.}
\end{aligned}
$$

which is in complete agreement with experiment. 
The quantum concept was also used by Einstein in 1905 to explain the photoelectric effect. Einstein postulated that the quanta of radiation of energy, $\mathrm{E}=\mathrm{h} \mathrm{v}$ behaves as particles called photons. From the special theory of relativity, the photon rest mass must be zero as it travels with the velocity of light c. Then the relativistic relation between enegy \& momentum is ;

$\mathrm{E}^{2}=\mathrm{p}^{2} \mathrm{c}^{2}+\mathrm{m}^{2} \mathrm{c}^{4}$ Since, $\mathrm{m}=0$

$\Rightarrow \mathrm{E}^{2}=\mathrm{p}^{2} \mathrm{c}^{2} \Rightarrow \frac{\mathrm{E}}{\mathrm{C}}=\mathrm{p}=\frac{\mathrm{h} v}{\mathrm{c}}=\frac{\mathrm{h}}{\lambda}, \lambda=$ wavelength.

Einstein used this idea of the corpuscular nature of radiation to explain the photoelectric effect. If the light of frequency $v$ falls on the surface of a metal, electrons come out for h $v>\phi$, where $\phi=$ work function of the metal but there is no photoelectron emission if $h v<\phi$. The energy of electron is, $E_{e}$ $=h \mathrm{v}-\phi$ is the Einstein photo electric law \& is in agreement with the experiment. Here, $\mathrm{E}_{\mathrm{e}}$ does not depend on the intensity of radiation but only on its frequency $v$. This cannot be explained by classical theory based on continuous exchange of energy.

According to the classical electromagnetic theory, there is a time lag between the fall of photon on the target and the emission of the photoelectron, in photoelectric effect which is in contrary to fact that, there is no time lag between the fall of photon on the target and the emission of the photoelectron as explained by Quantum theory. Consider an example of visible light falling on sodium surface gives a detectable photoelectric current if the incident intensity is about $10^{-6} \mathrm{watt} / \mathrm{m}^{2}$. A layer of sodium of one atom thickness and $1 \mathrm{~m}^{2}$ area has about $10^{19}$ atoms. So, assuming that the incident energy is received by the upper most atoms only, the average energy received by each atom is $10^{-25}$ watt. The ionization potential is about $4 \mathrm{eV}$. So, according to electromagnetic theory, the time required for an electron to get enough energy (i.e., $4 \mathrm{eV}$ ) to escape out of the sodium atom by photoelectric effect is $\mathrm{t}=\frac{4 \times 1.6 \times 10^{-19}}{10^{-25}} \sec 74$ days. Which is in contrary to the fact that, in photoelectric effect there is no time lag between the fall of photon on the target and the emission of the photoelectron.
In 1924Comptondemonstrated theparticlesbehaviour of radiation. He studied the scattering of x-rays by free or weakly bound electrons. When a photon collides with an electron at rest then we obtain the Compton formula $\Lambda \lambda=\lambda_{2}-\lambda_{1}=\left(\frac{2 \mathrm{~h}}{\mathrm{mc}}\right) \sin ^{2} \theta / 2$

where, $\Lambda \lambda=$ change in wave length, $\theta$ is the angle which the scattered photon makes with respect to the direction of the incident photon. The above formula agrees with the experiments. This result follows if the photon is treated as a particle with both momentum and energy. Classically, this wave length shift, which is proportional to the Planck Constanth, is not obtained but proportional to the radiation intensity. Hence, classical mechanics cannot explain the Compton effect. There consideration show that the electromagnetic radiation exhibits wave-particle duality.

The classical mechanics fails to explain the stability of atom well as the atomic emission and absorption spectra as they consists of narrow lines. But they are easily explained in the quantum theory when atoms emit or absorbs photons of well-determined frequencies i.e., energies. Only certain discrete energy values $E_{n} n=1,2 \ldots \ldots$ are allowed for the atom \& the frequency of the emitted or absorbed photon is given by, $h v_{m n}=\left|E_{m}-E_{n}\right|$. Niels Bohar in 1913 assumed that the electron occupies circular orbits around the nucleus in a hydrogen atom. The allowed orbit is one for which the angular momentum, 1 of the electron is an integral multiple of $\hbar$ i.e., $1=\mathrm{n} \hbar, \mathrm{n}=1,2 \ldots \ldots$ This at once lead the discrete values of the energy En, which is impossible from classical mechanics.

In 1923, De-Broglie put a hypothesis which states that material particle can have a wave like aspect described by $\mathrm{P}=\mathrm{h} / \lambda \Rightarrow \lambda=\frac{\mathrm{h}}{\mathrm{p}}$

The De-Broglie relation links the particle variable $p$ with wave variable $\lambda$ and is applicable both to radiation $\&$ matter. In this sense, the quantum theory unifies two concepts which were different from the classical.

Thus a dual character of electromagnetic radiation that it behaves as waves \& particles became evident from quantum concept. And, now a days the quantum physics has become indispensable for the researchers in physics. वण 